\title{
Suppression dampens unpleasant emotion faster than reappraisal: Neural dynamics in a Chinese sample
}

\author{
YUAN JiaJin*, LONG QuanShan, DING NanXiang, LOU YiXue, LIU YingYing \\ \& YANG JieMin \\ Key Laboratory of Cognition and Personality (SWU), Ministry of Education, Chongqing 400715, China; School of Psychology, Southwest \\ University, Chongqing 400715, China
}

Received May 4, 2014; accepted June 7, 2014; published online October 13, 2014

\begin{abstract}
The timing dynamics of regulating negative emotion with expressive suppression and cognitive reappraisal were investigated in a Chinese sample. Event-Related Potentials were recorded while subjects were required to view, suppress emotion expression to, or reappraise emotional pictures. The results showed a similar reduction in self-reported negative emotion during both strategies. Additionally, expressive suppression elicited larger amplitudes than reappraisal in central-frontal P3 component (340-480 ms). More importantly, the Late Positive Potential (LPP) amplitudes were decreased in each $200 \mathrm{~ms}$ of the 800-1600 ms time intervals during suppression vs. viewing conditions. In contrast, LPP amplitudes were similar for reappraisal and viewing conditions in all the time windows, except for the decreased amplitudes during reappraisal in the 1400-1600 ms. The LPP (but not P3) amplitudes were positively related to negative mood ratings, whereas the amplitudes of P3, rather than LPP, predict self-reported expressive suppression. These results suggest that expressive suppression decreases emotion responding more rapidly than reappraisal, at the cost of greater cognitive resource involvements in Chinese individuals.
\end{abstract}

\section{Event-related potentials, expressive suppression, unpleasant emotion, cognitive reappraisal}

Citation: Yuan JJ, Long QS, Ding NX, Lou YX, Liu YY, Yang JM. Event-related potentials, expressive suppression, unpleasant emotion, cognitive reappraisal. Sci China Life Sci, 2015, 58: 480-491, doi: 10.1007/s11427-014-4739-6

The ability to regulate unpleasant emotion is important for human life in the changing environments [1-3]. According to the time points in which a strategy has its primary impact in the emotion-generative process, Gross and coworkers distinguished between antecedent-focused and responsefocused strategies [4,5]. The former refers to strategies such as cognitive reappraisal, which requires interpreting emotion stimuli in a detached, emotion-irrelevant manner to modify emotion responses before they are fully blown. Conversely, the latter involves strategies that modulate emotional responses through modifying emotion-expressive behaviors, at the late stage of emotion activity. A typical example of response-focused strategy is expressive sup-

*Corresponding author (email: yuanjiajin168@126.com; yuanjiaj@swu.edu.cn) pression [5,6]. Many studies revealed that cognitive reappraisal is effective in decreasing self-reported unpleasant emotion states, emotion-expressive behaviors and in reducing neural activity in the limbic brain system including amygdala and nucleus accumbens [4,7-9]. In contrast, it was reported that expressive inhibition was ineffective in decreasing the subjective experience of negative emotions such as anxiety [2,4,10-12], while significantly increased peripheral physiological responding and limbic system activation $[4,6,13]$.

In the early studies by Gross and colleagues, subjects who were presented with negative stimuli (e.g. films, pictures) received reappraisal, suppression or attending instructions. The results showed that reappraisal resulted in less negative experience, less emotion-expressive behaviors and 
decreased sympathetic physiological responding. In contrast, expressive suppression did not change subjective emotional experience, but increased emotion-related peripheral physiological responding (e.g. skin conductance level and finger temperature) and impaired verbal memory, despite reduced expressive behaviors $[4,10,14]$. In addition, using emotion-provoking film clips, Campbell-Sills and colleagues [2-3] investigated the association of emotion regulation styles and negative emotion levels in patients of anxiety and mood disorders. The results showed that higher levels of suppression were associated with increased negative emotion levels during both film delivery and post-film recovery periods [2-3]. Furthermore, Hofmann and colleagues [12] directly compared the behavioral and physiological indexes of anxiety regulation using expressive suppression and cognitive reappraisal during an impromptu speech task. The results showed that expressive suppression was associated with greater increases in both heart rate and subjective anxiety in comparison with cognitive reappraisal [12]. More recently, several studies with American subjects reported that suppressing the expression of negative emotions was associated with decreased wellbeing and increased depressive symptoms $[15,16]$.

However, as a social species, the human lives in various interpersonal relations. It is common and adaptively important, in life settings, for people to regulate negative and other maladaptive emotions by inhibiting emotionexpressive behaviors, not only for keeping normal interrelation with other people, but also for the avoidance of violence, impulsive behavior, and other socially undesirable conducts [17]. This feature is more noticeable in East Asian cultures, whose collectivistic cultural norms highlight the avoidance of hurting others, and the efforts to preserve and experience relational harmony [18-21]. Though many studies showed evidences that expressive suppression was less effective in reducing negative emotion than reappraisal [2-4,10-12], these evidences, to date, unanimously came from studies conducted in western cultural backgrounds.

In fact, a growing number of recent studies indicated that the efficacy of expressive suppression in dampening negative emotions is culture-specific; such that expressive suppression is associated with better social functioning in East Asian cultures [18-21]. For instance, Butler and colleagues [18] investigated cultural influences on the efficacy of expressive suppression in dampening unpleasant emotion. The results showed that expressive suppression was associated with adverse psychological functioning for European Americans, but not for Chinese participants. In addition, it was reported that habitual suppression of emotion expression was associated with greater self-protections and higher negative emotions in European Americans [18]. However, the suppression was associated with decreased negative emotions in Asian Americans, corroborating enhanced efficacy of expressive suppression in dampening negative emo- tions in eastern culture [18]. These findings were confirmed by a recent ERP study, showing that emotion suppression, compared to attending instruction, was linked with reduced late positive potentials to negative pictures in Asian but not in European Americans [22]. In line with these evidences, a functional MRI study revealed that, in contrast to the significant negative mood induced by attending to negative pictures, the mood state did not change significantly after negative stimulation during expressive suppression in Japanese subjects. Also, the effectiveness of expressive suppression in dampening negative emotions was verified by a recent study in Hong Kong which showed less negative emotions and higher sales productivity as a function of increased suppression in insurance workers [23].

All these evidences suggest that expressive suppression is effective in reducing negative experiences and improving social functioning in Asian cultures. Thus, the prior findings from western subjects that expressive suppression is less useful in regulating unpleasant emotion in comparison with reappraisal $[4,6,10]$ may not apply to Chinese subjects. Instead, based on these abundant evidences, it is likely that expressive suppression is as effective as, or even more effective than, reappraisal in decreasing negative emotion in Chinese individuals.

Furthermore, an important part of evidences that support less regulation efficacy with suppression versus reappraisal was that expressive suppression elicited enhanced peripheral physiological responding (e.g. enhanced Skin Conductance, Heart Rate) and greater limbic activations (insula, amygdala or orbitofrontal cortex) in comparison with reappraisal $[4,6,11,13]$. However, as indicated by quite a few studies, the increased peripheral physiological and limbic area activations were not necessarily a result of emotional arousal $[13,24]$. These activations may also result from increased cognitive load during cognitive-demanding tasks such as working memory tasks [24,25], mental arithmetic task [26] or voluntary emotional suppression [13]. Thus, it is highly necessary to explore the efficacy of emotion regulation by suppression and reappraisal with a high temporal resolution technique, which is able to isolate the index of cognitive load from that of emotion arousal.

Apparently, Event-Related brain Potential (ERP) technique fits this purpose. On the one hand, expressive suppression is a resource-costly strategy that requires effortful monitoring of prepotent emotion-expressive behaviors $[4,10,13]$. Previous studies reported that suppressing prepotent behaviors was linked with a larger central-frontal P3 peaking around $400 \mathrm{~ms}$ post stimulus (e.g. No-go P3; $[27,28])$, which was suggested to reflect enhanced cognitive resources engaged in inhibitory processing [28-30]. This monitoring, however, was absent for the strategy of cognitive reappraisal $[4,10]$. Therefore, it is likely that expressive suppression elicits enhanced central-frontal P3 amplitudes compared to reappraisal. Though there was an ERP study that compared emotion regulations using suppression and 
reappraisal [31], the authors did not examine inhibitory-relevant components (e.g. central-frontal P3), thus unable to isolate emotional arousal from inhibitory process in brain potentials.

On the other hand, many studies have revealed that Late Positive Potential (LPP), a posterior-parietal positive slow ERP that reaches its largest amplitudes 500-700 ms post-stimulus and lasts for several hundred milleseconds, was more pronounced for emotionally salient than for neutral stimuli [9,32-36]. Moreover, LPP amplitude, which has been accepted an ideal index for the intensity of emotion experience: the LPP amplitudes decrease with the reduction of emotion experience during emotion regulation [9,32-34]. Thus, the LPP in brain potentials is a proper index for studying the temporal dynamics of emotion arousal during expressive suppression and cognitive reappraisal.

Based on the above analyses, we can see that it is possible to isolate the index of expressive inhibitory processing from that of late emotional reactivity by ERP measures. Specifically, expressive inhibitory processing might be associated with enhanced P3 amplitudes in central-frontal regions [28-30]; while the timing features of emotional responding is probably reflected by the LPP amplitudes varying as a function of regulation strategy [9,33,34]. Therefore, we predict that subjective experience of negative emotion is positively correlated with LPP amplitudes, and the self-reported levels of suppression should be positively related to central-frontal P3 amplitudes during expressive suppression. More importantly, as expressive suppression engages greater cognitive resources relative to reappraisal [10] and that suppressing the display of negative emotions is valued by Chinese cultural norms [18,21]; we predict that expressive suppression decreases negative emotion more quickly than reappraisal in Chinese individuals. Specifically,

because LPP amplitude is a valid index of emotion arousal levels, we predict that the LPP amplitude reduction might happen at an earlier time point during suppression compared to reappraisal in Chinese subjects.

\section{Materials and Methods}

\subsection{Subjects}

As paid volunteers, 18 (19-23 years; M=20.82 years, 9 males) students from Southwest University in China participated in the experiment. The subjects reported no history of affective disorder and were free of any psychiatric medication. The subjects were affectively healthy, indicated by the low scores in the Spielberger state-trait anxiety scale (STAS; total $=80$ for either scale) and Beck depression inventory (BDI; total=63). The averaged trait, state anxiety and depression scores were 38.06 (S.E.=1.91), 34.62 (S.E.=1.76), and 13.38 (S.E.=2.07), respectively. The EEG data of two subjects (1 male) were rejected from offline ERP analysis due to insufficient artifact-free trials available for ERP averaging. The subjects were right-handed, had normal or corrected to normal vision. The remaining sixteen subjects were similar in the habitual use of cognitive reappraisal $(\mathrm{M}=18.87)$ and expressive suppression $(\mathrm{M}=16.63$; $\mathrm{t}(15)=1.50, P=0.16)$ in the Emotion Regulation Questionnaire (ERQ; [37] $)^{1)}$. The study was approved by the local Review Board for Human Participant Research and each subject signed an informed consent form prior to the experiment.

\subsection{Stimuli and procedures}

The present study used a block-design picture viewing task. The task consisted of 4 blocks, and each block included 40 picture stimuli that were taken from International Affective Picture System (IAPS) and its Chinese adapted Version (Chinese Affective Picture System-CAPS; [38,39]). The picture stimuli were neutral, emotionally irrelevant in the first Block, as a non-emotional baseline for computing emotion effect in later conditions (Neutral-View, NV). In the second Block, subjects were required to view 40 unpleasant pictures without using any emotion regulation strategies (Unpleasant-View, UV). Then, the last two blocks each required subjects to view 40 unpleasant pictures while regulating unpleasant emotion using expressive suppression (Unpleasant-Suppression, US), or cognitive reappraisal (Unpleasant-Reappraisal, UR) strategy, respectively. The order of the US and UR blocks was counterbalanced across subjects. The purpose of preceding regulation blocks with non-regulation blocks was to avoid any carry-over influence of regulation strategies on the subsequent non-regulation viewing condition, as recommended by Moser et al., [34], Gross [10] and Ohira et al. [13]. Unpleasant pictures were composed of the scenes of frightening animals, human attack and body mutilations while neutral pictures depicted the scenes of neutral animals and human activities ${ }^{2}$.

In order to avoid emotional habituation or sensitization when a single set of pictures are presented repeatedly, the currents study randomly selected three different sets of unpleasant pictures for the UV, US and UR conditions. Also, there was evidence showing a cultural bias when IAPS was applied to Chinese subjects [40]. In order to control these influences and attribute differences in dependent variables

1) ERQ is a 10 item, 7-point questionnaire designed for measuring habitual use of expressive suppression and cognitive reappraisal. The ERQ had no reversed scoring items. Because the ERQ contained 6 items for reappraisal and 4 items for suppression, we multiplied the original reappraisal scores with $4 / 6$, consequently to obtain reappraisal scores equivalent to the sum of 4 items. Therefore, the comparison was conducted between the original suppression scores and the converted reappraisal scores to exclude the influence of the number differences.

2) The experimental materials used for this study are available by contact to the corresponding author. 
solely to emotion regulation, firstly we need to conduct a separate procedure checking whether the three sets of pictures were similar in emotional parameters for Chinese people. For this purpose, four trained judges (two males) blind to research purposes were invited to rate the valence (9-point scale, from 1: extremely unpleasant to 9: extremely pleasant) and arousal (9-point scale, from 1: very calm to 9: very excited) of the pictures. The four judges were highly consistent in assessing the emotionality of the pictures. The inter-rater reliability (kendall' s coefficient of concordance) was $0.83\left(\chi^{2}(3)=9.90 ; P<0.05\right)$ for both valence and arousal dimensions. Therefore, we averaged the rating data across the four judges for each picture, and then conducted a one-way ANOVA for the valence and arousal of pictures with experimental condition as a predictor. The condition effect for arousal was highly significant $(\mathrm{F}(3,156)=242.31$, $P<0.001)$. The pariwise comparisons showed significantly higher arousal values for UV (M=7.20), UR (7.13) and US (6.97) conditions than the NV condition (3.03; all $P<0.001)$. In addition, the arousal values were not significantly different during the UV, UR and US conditions $(P=0.21$ for UV-US; 0.71 for UV-UR; and 0.38 for US-UR comparisons; uncorrected; see Figure 1A). Similarly, the condition effect for valence was also significant $(\mathrm{F}(3,156)=237.78, P<$ 0.001). The pariwise comparisons showed significantly lower valence values for UV (1.73), UR (1.76) and US (1.83) conditions in comparison with the NV condition (4.71; all $P<0.001)$. In addition, the valence values were not significantly different during the UV, UR and US conditions $(P=0.43$ for UV-US; 0.82 for UV-UR; and 0.58 for US-UR comparisons, uncorrected; see Figure 1B). Thus, the pictures used for UV, US and UR conditions were valid in inducing unpleasant emotion, and the emotion attributes of the pictures were kept similar across the three unpleasant conditions.

Subjects were seated in a quiet room at approximately $150 \mathrm{~cm}$ from a computer screen with the horizontal and vertical visual angles below $6^{\circ}$. Prior to each block, subjects were instructed of the task and were presented with 10 trials for practice. In block 1 and 2 , each trial was initiated by a $1000 \mathrm{~ms}$ presentation of a word "view", reminding subjects of the task in the block. The offset of the word was followed by a small black fixation cross on the white computer screen for $300 \mathrm{~ms}$. Then, a 300-700 ms blank screen was presented and was followed by the onset of pictures for $2000 \mathrm{~ms}$. Subjects were instructed to do nothing but to simply view and pay close attention to each picture stimulus. The interval between the offset of the picture and the next word stimulus was $1000 \mathrm{~ms}$. Between blocks; two mins of rest, which was the maximal time used by another 10 subjects to rest in a pilot study, were used for subjects to recover their mood to the baseline level.

In block 3 and 4, the stimulus stream was the same as that of block 1 and 2, except that the word changed into "suppression" or "reappraisal", for reminding subjects to use the corresponding strategy to regulate unpleasant emotion in that block. Participants were trained of the suppression and the reappraisal strategies during practice trials. Reappraisal instructions trained subjects to think of pictures objectively; for example, to regard themselves as detached observers and that the event has no personal relevance to them [41]. Suppression instructions trained participants to intentionally suppress the expression of emotion responses to pictures, by keeping their facial expressions unchanged so that someone watching their face was unable to detect what was being experienced subjectively. At the end of each block, subjects were required to rate their mood state by a self-report 7-point scale (1: neutral, non-emotional to 7: extremely unpleasant). Also, they were asked to rate how successful they suppressed emotion-expressive behaviors /reappraised the meanings of the pictures, at the end of suppression or reappraisal block by a 7-point scale (1: not successful at all; 7: completely successful).

\subsection{ERP recording and analysis}

The EEG was recorded from 64 scalp sites using tin electrodes mounted in an elastic cap (Brain Products), with the references on the left and right mastoids (average mastoid reference, [42]) and a ground electrode on the medial frontal aspect. The vertical electrooculograms (EOGs) were recorded supra- and infra-orbitally at the left eye. The horizontal EOG was recorded from the left versus right orbital rim.
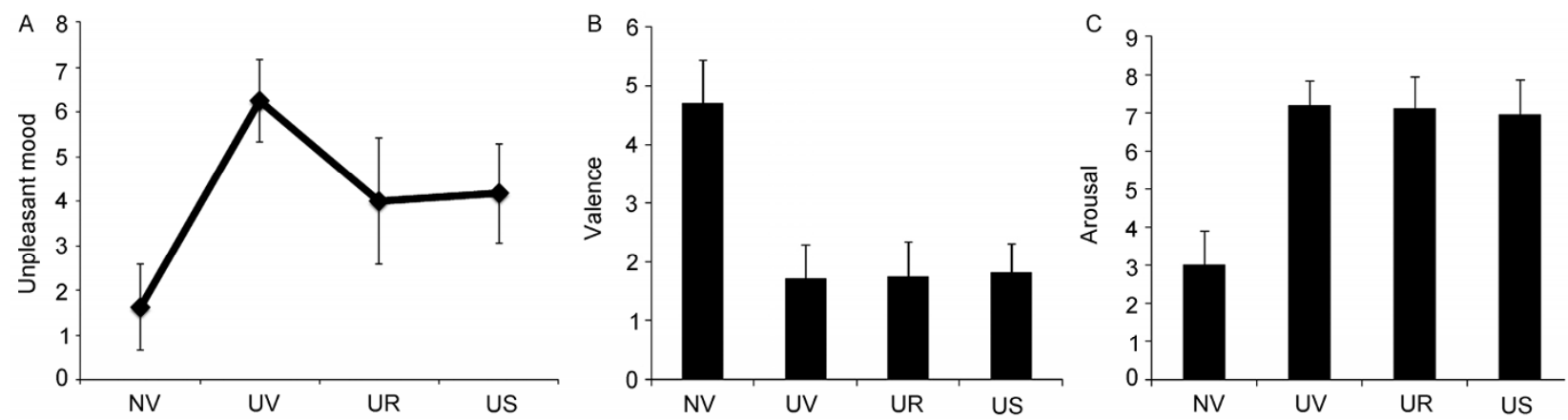

Figure 1 The results of mood rating for each block (A), and the results of valence (B) and arousal (C) assessment for pictures in each block (right). Error bars: \pm SD. 
The EEG and EOG were amplified using a DC 100 Hz bandpass and continuously sampled at $500 \mathrm{~Hz} / \mathrm{channel}$. All inter-electrode impedance was maintained below $5 \mathrm{k} \Omega$. Averaging of ERPs was computed off-line. Eye movement artifacts (blinks and eye movements) were rejected offline and $24 \mathrm{~Hz}$ low pass filter was used. Trials with EOG artifacts (mean EOG voltage exceeding $\pm 80 \mu \mathrm{V}$ ) and those contaminated with artifacts due to amplifier clipping, peak-to-peak deflection exceeding $\pm 80 \mu \mathrm{V}$ were excluded from averaging. Rejected trials were rare. There were on the average 38.0 trials for NV, 37.8 trials for UV, 38.4 trials for US and 37.6 trials for UR conditions obtained for ERP averaging $(\mathrm{F}(3,45)=0.83$, ns $)$.

The EEG in each block was averaged separately. The ERP waveforms were time-locked to the onset of stimuli and the averaged epoch for ERPs was $2200 \mathrm{~ms}$ including a $200 \mathrm{~ms}$ pre-stimulus baseline. As shown by Figure 2, each block elicited a prominent P2 in the 140-200 ms, and a prominent P3 component in the 340-480 ms interval across frontal and central scalp areas ${ }^{3)}$, consistent with prior studies involving prepotent behavioral inhibition [27,28]. Moreover, prominent Late Positive Potential (LPP) activity, which started from $600 \mathrm{~ms}$ and lasted until the offset of picture presentation (i.e., $2000 \mathrm{~ms}$ ), was observed at the posteriorparietal scalp region during each block (Figure 2), consistent with the topographical distributions in many prior studies [32-36]. Therefore, the amplitudes (baseline to peak) and peak latencies of P2 (140-200 ms), and the averaged amplitudes of P3 (340-480 ms) were measured and analyzed at the central and frontal areas (9 sites: Fz, F3, F4, FC3, FC4, FCz, Cz, C3, C4). We measured averaged amplitudes, instead of peak amplitudes, for P3 because the peak was not prominent in the NV condition. Moreover, the LPP amplitudes were measured in 600-2000 ms time windows at parietal region (9 sites: $\mathrm{CP} 3, \mathrm{CPz}, \mathrm{CP} 4, \mathrm{P} 3, \mathrm{Pz}, \mathrm{P} 4$, PO3, POz \& PO4).

A repeated-measure ANOVA was conducted on the latencies (P2) and the amplitudes (P2, P3 and LPP) of these components with Block [4 levels: NV, UV, US \& UR], saggittal (three levels: F, FC and C for P2 and P3; CP,P \& PO for LPP) and coronal (three levels: left, midline, right) as factors. In order to analyze the timing dynamics of LPPs during different instructions, LPP amplitudes in the 600-2000 ms were segmented into 7 consecutive time windows of $200 \mathrm{~ms}$ each), and timing (7 levels) was then submitted into the ANOVA as an independent variable. If a significant main effect of block or block by timing interaction was detected, the post hoc analyses were planned to focus on 1), testing the significance of the emotion effect (UV vs. NV) and the regulation effect (UV vs. US; UV vs.
UR); 2), testing how the timing of the emotion regulation effect in LPP amplitudes varied as a function of strategy. The degrees of freedom of the F-ratio were corrected according to the Greenhouse-Geisser method for any violations of sphericity, and Bonferroni-Holm method was used to adjust the $P$-value during post hoc pairwise comparisons if significant main or interaction effects were detected [31].

\section{Result}

\subsection{The mood assessment}

The analysis of mood rating data (based on a 7-point Likert scale) showed a significant main effect of block $\left(\mathrm{F}(3,45)=53.18, P<0.001 ; \eta 2_{\mathrm{p}}=0.78\right)$. The unpleasant mood rating during UV condition (6.25) was significantly higher than during NV condition (1.63). In addition, both UR and US conditions showed decreased unpleasant rating than the UV condition (both $P<0.001$ ), while the mood rating during reappraisal (4.00) was not significantly different from that during suppression (4.19; $P=0.66$; see Figure $1 \mathrm{~A})$.

\subsection{Manipulation check}

The analysis of the instruction conformation data (i.e. responses to the question " how successful did you reappraise the stimulus/suppress the expression of emotion?") showed that the reappraisal strategy was successfully used during the UR block (6.31) and the suppression strategy was successfully used during the US block (6.69). The scores were significantly higher than the midpoint of the rating scale (i.e.4) during both reappraisal $(\mathrm{t}(15)=9.12, P<0.001)$ and suppression $(\mathrm{t}(15)=22.46, P<0.001)$ blocks. The rating was not significantly different between suppression and reappraisal blocks $(\mathrm{t}(15)=1.57, P>0.10)$.

\subsection{The emotion effects in ERPs}

P2. The analysis of $\mathrm{P} 2$ amplitudes showed a significant main effect of block $(\mathrm{F}(3,45)=7.74 ; P=0.001)$. The amplitudes were smaller during neutral $(3.72 \mu \mathrm{V})$ compared to unpleasant blocks, irrespective of regulation strategies $\left(\mathrm{p}_{\mathrm{s}}<0.02\right)$. There were no significant differences across UV $(5.39 \mu \mathrm{V})$, US $(5.83 \mu \mathrm{V})$ and UR $(5.42 \mu \mathrm{V})$ conditions $\left(\mathrm{p}_{\mathrm{s}}>0.90\right)$. The analysis of P2 latencies did not yield any significant main or interaction effects.

P3. There were significant main effects of block $(\mathrm{F}(3,45)=9.82, P<0.001)$ and saggitality $(\mathrm{F}(2,30)=27.76$, $P<0.001)$. Central sites $(1.80 \mu \mathrm{V})$ recorded larger amplitudes than frontal sites $(-1.43 \mu \mathrm{V})$. The P3 amplitudes were

\footnotetext{
3) Figure 2 shows that $\mathrm{P} 3$ component was noticeable across both frontal, central and parietal sites. We conducted a preliminary analysis of emotion regulation effect in $\mathrm{P} 3$ amplitudes at $\mathrm{Fz}, \mathrm{FCz}, \mathrm{Cz}, \mathrm{CPz}$ and $\mathrm{Pz}$ sites, separately. The results showed that the regulation effect was significant in $\mathrm{Cz}$ and more anterior sites $\left(\mathrm{F}(2,30)=3.71-4.39 ; \mathrm{p}_{\max }=0.044\right)$ regions but not in $\mathrm{CPz}$ and $\mathrm{Pz}\left(\mathrm{F}_{\max }(2,30)=1.90, \mathrm{p}_{\min }=0.17\right)$. Thus, our analysis of $\mathrm{P} 3$ component just focused on central and more anterior regions.
} 

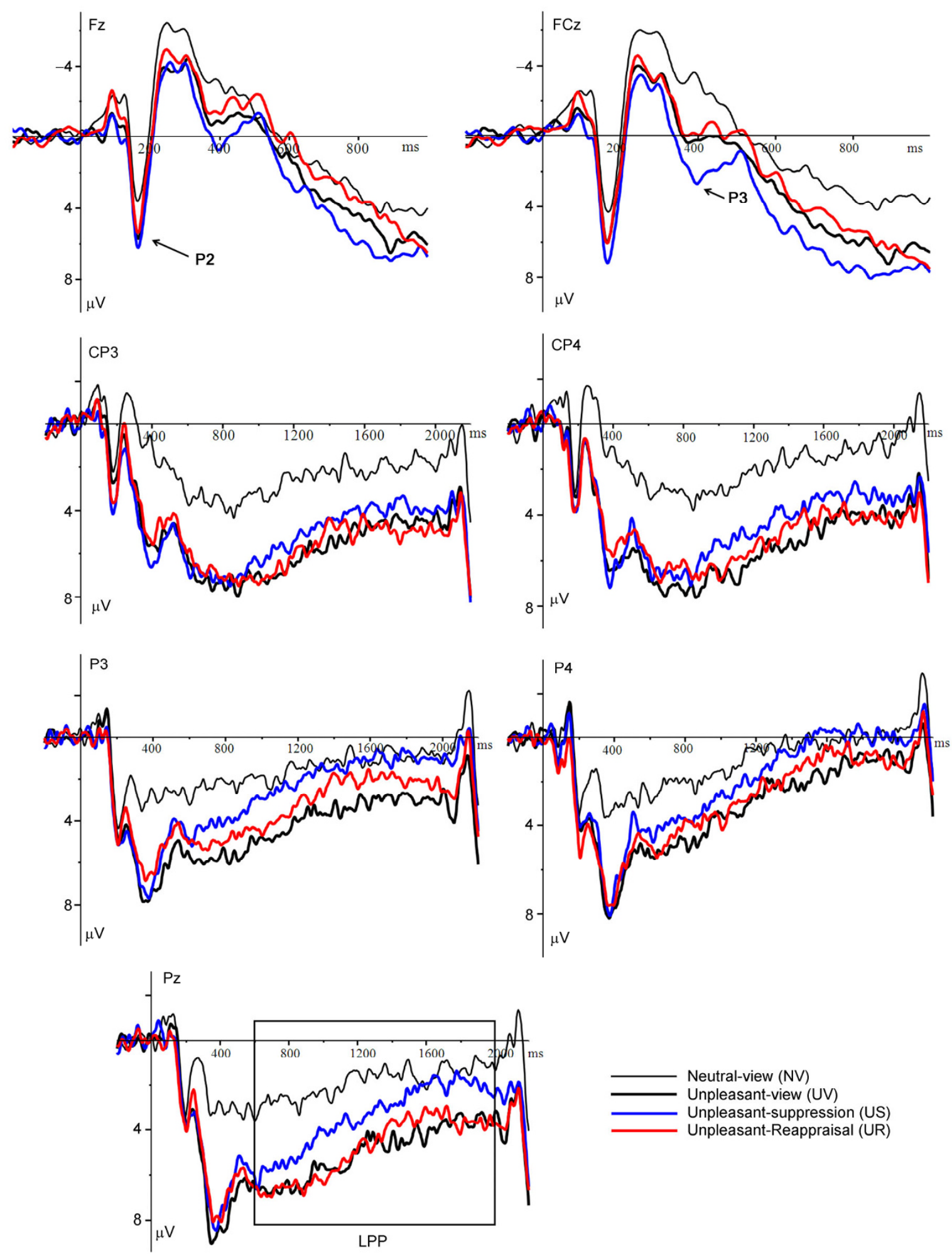

Figure 2 Averaged ERPs elicited by NV, UV, US, UR conditions at Fz, FCz, CP3, CP4, P3, P4 and Pz.

smaller for neutral $(-1.79)$ compared to negative pictures during UV $(0.65 \mu \mathrm{V} ; P<0.001)$, US $(1.71 \mu \mathrm{V} ; P<0.001)$ and of a smaller size, during UR $(-0.22 \mu \mathrm{V} ; P=0.045)$ conditions. In addition, US block exhibited larger P3 amplitudes compared to UR block $(\mathrm{F}(1,15)=8.72, P=0.03)$ which, however, displayed similar amplitudes with the UV condition $(\mathrm{F}(1,15)=1.55, P=0.69)$. No other effects were detected in this component. 


\section{Late Positive Potential (LPP)}

The LPP amplitudes were more pronounced at centroparietal $(4.82 \mu \mathrm{V})$ compared to parietal $(2.81 \mu \mathrm{V})$ or occipitoparietal $(-0.30 \mu \mathrm{V})$ sites $(\mathrm{F}(2,30)=37.16, P<0.001)$. There was a significant main effect of block $(\mathrm{F}(3,45)=11.60, P<0.001)$ and timing $(\mathrm{F}(6,90)=14.15, P<0.001)$. Negative pictures elicited enhanced amplitudes than neutral $(0.92 \mu \mathrm{V})$ pictures, regardless of regulation instructions (all $P<0.01$ ). Regarding emotion regulation effects, the post hoc analysis identified smaller amplitudes for US $(2.43 \mu \mathrm{V} ; P=0.036)$, but not UR $(3.15 \mu \mathrm{V} ; P>0.10)$, compared to $\mathrm{UV}(3.71 \mu \mathrm{V})$ blocks.

Moreover, there was a significant timing by condition interaction $(\mathrm{F}(18,270)=2.70, P=0.023)$. In order to break down this interaction, we tested the main effect of block in each of the seven time windows, respectively. If the condition effect was significant, the post hoc pairwise comparisons focused on the UV-US and the UV-UR differences, similar to the approach used by Paul and colleagues [31].

600-800 ms: the main effect of block was significant $(\mathrm{F}(3,45)=11.89, P<0.001)$. Post hoc pairwise comparisons showed that this effect was due to the larger LPP amplitudes for negative versus neutral pictures, regardless of regulation instructions (all $P<0.01$ ). The LPP amplitudes were not significantly different across UV $(5.29 \mu \mathrm{V})$, US $(4.50 \mu \mathrm{V})$ and UR $(4.82 \mu \mathrm{V}$ ) blocks (all $P>0.50$ ).

800-1000 ms: the main effect of block was significant $(\mathrm{F}(3,45)=15.25, P<0.001)$. Post hoc pairwise comparisons showed smaller LPP amplitudes for US condition $(3.51 \mu \mathrm{V}$; $P=0.036)$, but not UR condition $(4.34 \mu \mathrm{V} ; P=0.27)$, in comparison with the UV condition $(4.89 \mu \mathrm{V})$.

1000-1200 ms: the main effect of block was significant $(\mathrm{F}(3,45)=13.10, P<0.001)$. Post hoc pairwise comparisons showed smaller LPP amplitudes for US condition $(2.50 \mu \mathrm{V}$; $P=0.024)$, but not UR condition (3.67 $\mu \mathrm{V} ; P=0.13$ ), in comparison with the UV condition $(4.29 \mu \mathrm{V})$.

1200-1400 ms: the main effect of block was significant $(\mathrm{F}(3,45)=8.18, P=0.002)$. Post hoc pairwise comparisons showed smaller LPP amplitudes for US condition $(1.54 \mu \mathrm{V}$; $P=0.026)$, but not UR condition $(2.50 \mu \mathrm{V} ; P=0.09)$, in comparison with the UV condition $(3.27 \mu \mathrm{V})$.

1400-1600 ms: the main effect of block was significant $(\mathrm{F}(3,45)=6.93, P=0.002)$. Post hoc pairwise comparisons showed smaller LPP amplitudes for both US condition $(0.93$ $\mu \mathrm{V} ; P=0.005)$ and UR condition $(1.73 \mu \mathrm{V} ; P=0.012)$ in comparison with the UV condition $(2.94 \mu \mathrm{V})$.

1600-1800 ms: the main effect of block was not significant $(\mathrm{F}(3,45)=1.47, P=0.24)$.

1800-2000 ms: the main effect of block was not significant $(\mathrm{F}(3,45)=2.18, P=0.12)$.

Thus, US condition elicited smaller LPP amplitudes compared to UV condition from $800 \mathrm{~ms}$ post picture onset, while UR condition did not produce the significant LPP reduction effect until $1400 \mathrm{~ms}$ post stimulus (Figure 3).

\subsection{Correlation analyses}

To test whether LPP amplitude is valid, as reported, in predicting the intensity of emotion experience, we computed spearman rank-order correlation between the LPP amplitudes and the scores of unpleasant mood rating, in the UV versus NV conditions whose emotion effect is free of the impact of regulation. The LPP amplitudes for correlation analyses were extracted from the 600-1600 ms, because this window showed a significant emotion effect. The results showed that the LPP amplitude effect was positively correlated with the intensity of unpleasant experience $(\mathrm{r}=0.571, P=0.010$; Figure 4A). To clarify whether this correlation was specific to LPP amplitudes, a similar correlation was computed between the mood effect and the amplitude effect in P3, another component that also involves late conscious processing [27]. This correlation, however, was non-significant ( $\mathrm{r}=0.056, P=0.418$; Figure 4B).

Because the LPP amplitude reduction was specific to expressive suppression in the $800-1400 \mathrm{~ms}$ interval, we also computed the spearman correlation between the emotion effect of LPPs in this interval and the negative mood levels during US compared to NV conditions. The result demonstrated a similar positive correlation between the two variables ( $r=0.443, P=0.043$; Figure $4 \mathrm{C}$ ), which reinforced the validity of LPPs in reflecting subjective negative emotion.

Lastly, to verify whether the pronounced P3 amplitudes during US condition reflects brain processing of suppressing emotion-expressive behaviors, an additional spearman correlation was computed between the suppression rating and the US-NV amplitude differences in P3. The result showed a trend of larger P3 amplitude enhancement from $\mathrm{NV}$ to US conditions with increasing suppression ratings ( $\mathrm{r}=0.407, P=0.059$; Figure 4D). This trend was specific to the P3, as we failed to observe a similar correlation between the LPPs and suppression rating ( $\mathrm{r}=0.094, P=0.365)$.

Taken together, these results suggested that the pronounced P3 during US condition was most likely an indicator of expressive inhibitory processing, while the subsequent LPP amplitude, as indicated by prior studies, was a valid index of the subjective experience of unpleasant emotion (Figure 4A-D).

\section{Discussion}

Using event-related potential technique, the present study investigated the timing dynamics of unpleasant emotion regulation by expressive suppression and cognitive reappraisal in a Chinese sample. The main findings demonstrated that 1), unpleasant pictures elicited greater unpleasant report, and greater positive amplitudes across P2, P3 and LPP components in comparison with neutral pictures, suggesting that our manipulation of negative pictures were successful; 2), reappraisal and suppression decreased sub- 

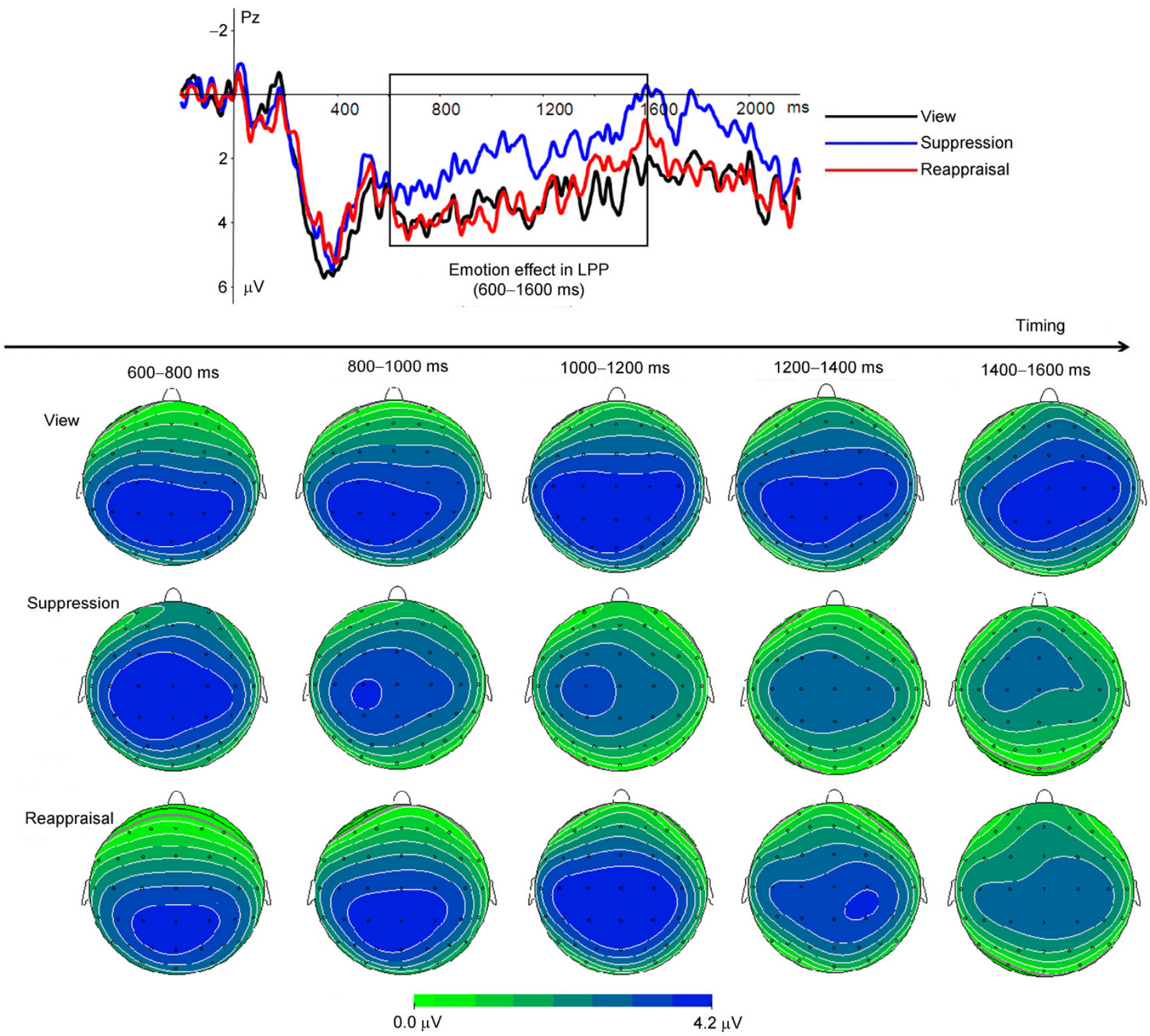

Figure 3 Top Panel: Unpleasant-neutral difference ERPs at Pz during viewing (black), expressive suppression (blue) and reappraisal (red) conditions. Bottom Panel: Topographical maps of the voltage amplitudes of unpleasant-neutral difference waves during viewing, suppression, and reappraisal conditions from 600 to $1600 \mathrm{~ms}$. It can be observed that the unpleasant effect reflected by LPP decreased at earlier time points during suppression vs. reappraisal.

jective negative emotion to a similar extent; 3 ), expressive suppression induced larger amplitudes compared to reappraisal in central-frontal $\mathrm{P} 3$, a component established to reflect response inhibitory processing during behavioral inhibition studies [27-29]; 4), the reduction of LPP amplitudes for negative pictures happened at earlier time points during suppression compared to reappraisal. These results suggest that expressive suppression dampens unpleasant emotion more quickly than cognitive reappraisal in Chinese individuals.

\subsection{Early components}

In the present study, the P2 component that peaked around $200 \mathrm{~ms}$ post stimulus, showed similar amplitudes during UV, US, UR conditions which, however, all elicited larger positive amplitudes than the NV condition. This coincided with prior reports of larger P200 amplitudes for emotional than for neutral stimuli that reflects enhanced attention allocation to biologically important stimuli $[43,44]$. The application of regulation strategies did not significantly influence P2 amplitudes, probably because this component was located in an early time point where conscious cognition is inaccessible [45,46].

US condition elicited larger P3 amplitudes than UR condition which, however, elicited similar P3 amplitudes as the UV condition across central and frontal sites. Previous studies have indicated that expressive suppression is a re- 

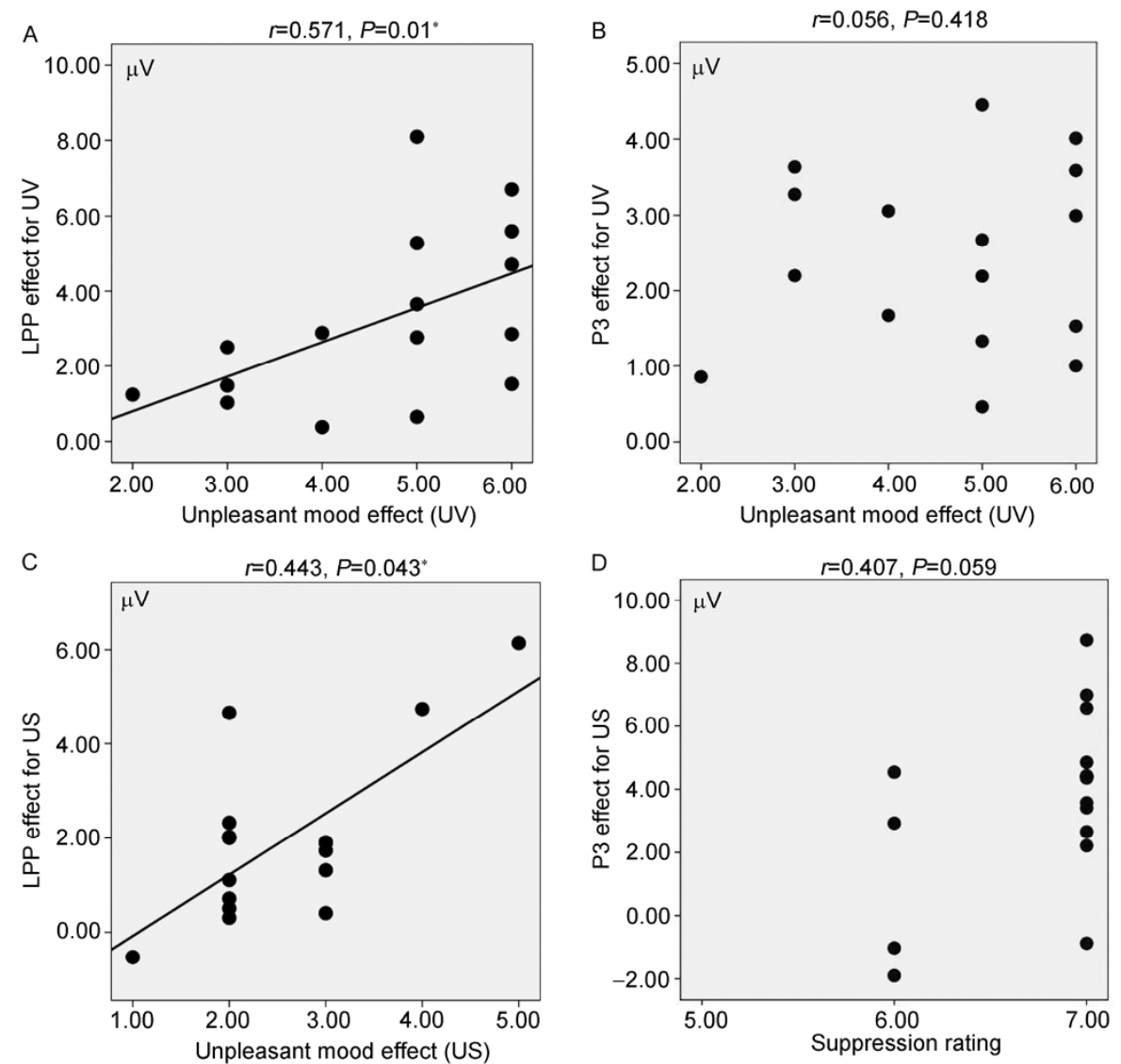

Figure 4 The scatterplot for the spearman rank-order correlation between the negative mood and the LPP effect (600-1600 ms) during UV vs. NV conditions (A); between the negative mood and the P3 effect during UV vs. NV conditions (B); between the negative mood and the LPP effect (800-1400 ms) during US vs. NV conditions (C); between the suppression rating and the P3 effect during US vs.NV conditions (D). *, $P<0.05$.

source-costly strategy that requires effortful monitoring of the prepotent emotion-expressive behaviors $[4,10,13]$. By contrast, it has been indicated that reappraisal is an economical strategy that does not require subjects to conduct effortful monitoring [10]. As indicated, suppressing prepotent behaviors was linked with a larger central-frontal P3 component peaking around $400 \mathrm{~ms}$ post stimulus (i.e. No-go P3; $[27,28]$ ), which reflects enhanced engagement of cognitive resources during inhibitory processing [28-30]. Therefore, the effortful inhibitory processing recruited during suppression instead of reappraisal most likely accounted for larger p3 amplitudes during suppression compared to reappraisal strategies. This interpretation was supported by our finding that the P3 amplitude enhancement during US vs. NV conditions positively predicts self-reported expressive suppression. On the other hand, the P3 amplitudes were similar during UR and UV conditions. This confirms that reappraisal is an economical strategy that does not engage much self-monitoring process $[4,6,10]$, as the $\mathrm{P} 3$ amplitudes were found to increase with greater resources mobilized for cognitive processing $[29,47]$.

\subsection{Emotion regulation effects in LPPs}

The present study observed prominent LPP activity starting from $600 \mathrm{~ms}$ and lasting until $2000 \mathrm{~ms}$ post stimulus. Consistent with prior reports $[35,36,48]$, LPP amplitudes were enlarged for unpleasant than for neutral stimuli in the $600-1600 \mathrm{~ms}$ during the viewing condition, and this amplitude enhancement was positively related to the subjective experience of negative emotion. This confirms that the LPP amplitude elicited by emotional stimuli is a valid index of subjective emotion arousal [32,33].

More importantly, we observed decreased LPP amplitudes for US relative to UV conditions, and this amplitude reduction began from $800 \mathrm{~ms}$ and sustained until $1600 \mathrm{~ms}$ post stimulus. In contrast, the LPP amplitudes were similar in the 600-1400 ms, and then decreased in the 1400-1600 ms time interval during UR compared to UV conditions. This suggests that expressive suppression reduced negative emotional responding more quickly compared to cognitive reappraisal. LPP amplitude modulation has proved to be an ideal index of the effect of emotion regulation, with the amplitude reduction positively related to the decrease in self-reported emotion [9,32-34]. Consistent with these re- 
ports, our results of correlation analysis reinforced the validity of LPP amplitude in reflecting the intensity of subjective emotion (Figure 4). Thus, our timing analysis of the LPP modulation effectively delineated the timing features of emotion downregulation, such as determining the time points for a strategy to take effect in reducing emotional reaction.

\subsection{Implications}

Taking P3 and LPP results together, the present study demonstrated that expressive suppression, which recruited more cognitive resources indexed by enlarged P3 activity, was faster in reducing the intensity of unpleasant emotion responding compared with cognitive reappraisal in Chinese individuals. This was in line with a number of recent studies, which indicated that Asian cultures more encourage the suppression of negative emotional expression in comparison with western cultures [1,18-21]. This is because the expression of negative emotions may hurt someone else or are detrimental to social and relational harmony, which is valued by interdependent and collectivistic cultural norms in Asia countries [1,20,21]. For example, Friesen [49] observed that Japanese and European participants showed the same facial expressions when watching an emotional film alone, but Japanese showed less negative expressions in the presence of experimenter. In addition, many studies reported greater emotion suppression in eastern cultures, such as the report of greater habitual suppression in Asian Americans [37], the report that Asian values discourage assertiveness and encourage self-regulation when interacting with people of higher social status [50], and that suppressing emotion displays was considered as normative in collectivistic nations [21,51]. Consistent with all these evidences, the current study observed that suppression is as effective as reappraisal in dampening experienced negative emotion. Furthermore, our findings extended these previous studies by showing that suppression down-regulated negative emotional reaction more quickly than reappraisal in Chinese. Thus, expressive suppression is not necessarily less effective than cognitive reappraisal in down-regulating negative emotion. Instead, this strategy is faster than reappraisal in regulating emotional reaction, at least, in a culture that values suppressing negative expressions.

However, expressive suppression was consistently reported to enhance physiological responding compared to reappraisal in heart rate, skin conductance response as well as activations in prefrontal and limbic cortices [4,6,13]. For instance, it was reported that disgust-expressive suppression significantly increased neural activity in limbic regions (e.g. amygdala and insula; [6]); and that suppressing emotion expression to IAPS pictures elicited larger skin conductance responses and greater orbitofrontal cortex activations [13]. Based on these findings, Researchers inferred that expressive suppression was a maladaptive strategy in terms of emotion regulation [4,6]. However, the increased peripheral-physiological and limbic activations were not necessarily a unique reflection of emotional impact [13,24]. These activations also resulted from increased cognitive load during resource-demanding tasks such as working memory task [24], or voluntary emotional suppression [13]. Despite this fact, no study to date has isolated the index of cognitive load from that of regulating emotion arousal during emotion-expressive suppression.

With high temporal resolution ERPs, the present study observed that central-frontal P3 amplitudes were larger during suppression compared to reappraisal conditions, and the P3 (but not LPP) amplitude showed a positive correlation with self-reported suppression. In contrast, LPP (but not P3) amplitudes were positively related to experienced negative emotion. These results showed that central-frontal P3 amplitudes were most likely an index of cognitive load during expressive suppression while LPP amplitude modulation, as consistently indicated [33,34], was a reflection of emotion arousal modulation during suppression. Therefore, it is likely that the enhanced physiological and limbic responding observed in prior studies reflects enhanced cognitive cost during expressive suppression, rather than emotional consequences that shared these patterns of activations. Considering that the speeded regulation during suppression was preceded by enhanced cognitive costs, future studies need to investigate emotion regulation by combing suppression and reappraisal, to explore the likelihood of enhancing regulating efficiency without much involvement of cognitive cost.

The present study preceded the regulation blocks with the viewing block to avoid potential confounds of practicing regulation strategies on the emotional arousal effects during the viewing condition. One may question that this design leads to emotional habituation from viewing to regulation blocks, which confounds the effects of emotion regulation. This possibility, however, may not be true based on the following two reasons. Firstly, the current study used three different sets of negative pictures for UV, UR and US conditions. Each condition included 40 pictures; and each picture was presented just for once in the experiment, which is in disagreement with the notion of emotional habituation in prior studies that examined the impacts of repeated stimulus presentation on one's reaction to emotional stimuli [52-54].

Secondly, there were a couple of evidences showing that the humans are resistant to emotional habituation during negative stimulation, whether the stimulus presentation was repeated [52) or unrepeated [55,56]. For instance, Carretié et al. [52] directly explored the emotional habituation effects using repeated stimulus presentation and ERP measures. The results showed that the N100 activity to positive and neutral stimuli was significantly reduced during the second compared to the first experimental phases. However, the N100 amplitudes were not significantly reduced in the second compared to the first phases during 
negative stimulation. In addition, using affective priming and an emotional stroop task, Smith and colleagues [55] observed that the color naming for negative words was prolonged compared to that for positive words, and this effect was reliable, unaffected by whether the emotional words were preceded by negative primes, or by no-prime. This suggests that the antecedent perception of negative information does not decrease the emotional effects for negative words. Recently, we investigated the impact of listening to positive and negative music on the brains' responding to emotional pictures. By computing emotional-neutral differences in LPP amplitudes as an index of emotional arousal to pictures, we observed that the emotional arousal to negative pictures was not decreased, but instead increased by listening to negative music before picture onset [56]. Based on these evidences, the LPP and negative emotion reductions during regulation compared to viewing blocks in the current study was most likely a result of emotion regulation, rather than emotional habituation.

\section{Conclusions}

Expressive suppression is as effective as cognitive reappraisal in down-regulating the intensity of experienced negative emotion. Furthermore, suppression dampens negative emotional responding more quickly than reappraisal in Chinese individuals, at the cost of greater involvement of cognitive resources. LPP is a unique index of emotional reaction while central-frontal $\mathrm{P} 3$ is an index of suppressing emotion-expressive behaviors in brain potentials.

This study was supported by National Natural Science Foundation of China (NSFC31170989;31371042), the Keygrant Project of Chinese Ministry of Education (NO. 311032), and the Special Grant for Postdoctoral Research in Chongqing (Xm2014059).

1 Wierzbicka, A. Emotion, language, and cultural scripts. In: Kitayama S. Markus HR, eds. Emotion and culture. Washington, DC: American Psychological Association, 1994: 133-196

2 Campbell-Sills L, Barlow DH, Brown TA, Hofmann SG. Acceptability and Suppression of Negative Emotion in Anxiety and Mood Disorders. Emotion, 2006, 6: 587-595

3 Campbell-Sills L, Barlow, DH, Brown TA, Hofmann SG. Effects of suppression and acceptance on emotional responses of individuals with anxiety and mood disorders. Behav Res Ther, 2006, 44: 1251-1263

4 Gross JJ. Antecedent- and response-focused emotion regulation: divergent Consequences for Experience, Expression, and Physiology. J Pers Soc Psychol, 1998, 74: 224-237

5 Gross JJ, Thompson RA. Emotion Regulation: conceptual foundations. Chapter 1, Handbook of Emotion Regulation, 2007: 3-24

6 Goldin PR, McRae K, Ramel W, Gross JJ. The neural bases of emotion regulation: Reappraisal and suppression of negative emotion. Biol Psychiatry, 2008, 63: 557-586

7 Ochsner KN, Bunge SA, Gross JJ, Gabrieli JD. Rethinking feelings: An fMRI study of the cognitive regulation of emotion. J Cogn Neurosci, 2002, 14: 1215-1229

8 Phan KL, Fitzgerald DA, Nathan PJ, Moore GJ, Uhde TW, Tancer,
ME. Neural substrates for voluntary suppression of negative affect: A functional magnetic resonance imaging study. Biol Psychiatry, 2005, 57: 210-219

9 Hajcak G, Nieuwenhuis S. Reappraisal modulates the electrocortical response to unpleasant pictures. Cogn Affect Behav Neurosci, 2006, 6: 291-297

10 Gross JJ. Emotion regulation: Affective, cognitive, and social consequences. Psychophysiology, 2002, 39: 281-291.

11 Harris CR. Cardiovascular responses of embarrassment and effects of emotional suppression in a social setting. J Pers Soc Psychol, 2001, 81: 886-897.

12 Hofmann SG, Heering S, Sawyer AT, Asnaani A. How to handle anxiety: The effects of reappraisal, acceptance, and suppression strategies on anxious arousal. Behav Res Ther, 2009, 47: 389-394

13 Ohira H, Nomura M, Ichikawa N, Isowa T, Iidaka T, Sato A, Fukuyama S, Nakajima T, Yamada J. Association of neural and physiological responses during voluntary emotion suppression. Neuroimage, 2006, 29: 721-733

14 Richards JM, Gross JJ. Emotion regulation and memory: The cognitive costs of keeping one's cool. J Pers Soc Psychol, 2002, 79: 410-424

15 Langner CA, Epel ES, Matthews KA, Moskowitz JT, Adler NE. Social hierarchy and depression: the role of emotion suppression. $\mathbf{J}$ Psychol, 2012, 146: 417-436

16 Hopp H, Rohrmann S, Hodapp V. Suppression of negative and expression of positive emotions: Divergent effects of emotional display rules in a hostile service interaction. European Journal of Work and Organizational Psychology, 2012, 21: 84-105

17 Davidson RJ, Putnam KM, Larson CL. Dysfunction in the Neural Circuitry of Emotion Regulation-A Possible Prelude to Violence. Science, 2000, 289: 591-594

18 Butler EA, Lee TL, Gross JJ. Emotion regulation and culture: Are the social consequences of emotion suppression culture-specific. Emotion, 2007, 7: 30-48

19 Matsumoto D, Yoo SH, Nakagawa S. Culture, emotion regulation, and adjustment. J Pers Soc Psychol, 2008, 94: 925-937

20 Trommsdorff G, Rothbaum F. Development of Emotion Regulation in Cultural Context: Regulating Emotions: Culture, Social Necessity, and Biological Inheritance/Marie Vandekerckhove(eds). Malden: Blackwell, 2008: 85-120

21 Soto JA, Perez CR, Kim YH, Lee EA, Minnick MR. Is expressive suppression always associated with poorer psychological functioning? A cross-cultural comparison between European Americans and Hong Kong Chinese. Emotion, 2011, 11: 1450-1455

22 Murata A, Moser JS, Kitayama S. Culture shapes electrocortical responses during emotion suppression. Soc Cogn Affect Neurosci, 2013, 8: 595-601

23 Yeung DY, Fung HH. Impacts of suppression on emotional responses and performance outcomes: an experience-sampling study in younger and older workers. J Gerontol B Psychol Sci Soc Sci, 2012, 67: 666-676

24 Schaefer A, Braver TS, Reynolds JR, Burgess GC, Yarkoni T, Gray JR. Individual differences in amygdala activity predict response speed during working memory. J Neurosci, 2006, 26: 10120-10128

25 Gianaros PJ, Van Der Veen FM, Jennings JR. Regional cerebral blood flow correlates with heart period and high-frequency heart period variability during working-memory tasks: implications for the cortical and subcortical regulation of cardiac autonomic activity. Psychophysiology, 2004, 41: 521-530

26 Tanida M, Sakatani K, Takano R, Tagai K. Relation between asymmetry of prefrontal cortex activities and the autonomic nervous system during a mental arithmetic task: near infrared spectroscopy study. Neurosci Lett, 2004, 369: 69-74

27 Albert J, López-Martín S, Carretié L. Emotional context modulates response inhibition: neural and behavioral data. Neuroimage, 2010, 49: 914-921

28 Yuan JJ, Meng XX, Yang JM, Yao GH, Hu L, Yuan H. The valence strength of unpleasant emotion modulates brain processing of behavioral inhibitory control: Neural correlates. Biol Psychol, 2012, 89: 
$240-251$

29 Donkers FC, van Boxtel GJ. The N2 in go/no-go tasks reflects conflict monitoring not response inhibition. Brain Cogn, 2004, 56: 165-176

30 Falkenstein M, Hoormann J, Hohnsbein J. ERP components in Go/NoGo tasks and their relation to inhibition. Acta Psychol(Amst), 1999, 101: 267-291

31 Paul S, Simon D, Kniesche R, Kathmann N, Endrass T. Timing effects of antecedent-and response-focused emotion regulation strategies. Biol psychol, 2013, 94: 136-142

32 Foti D, Hajcak G. Deconstructing Reappraisal: Descriptions Preceding Arousing Pictures Modulate the Subsequent Neural Response. J Cogn Neurosci, 2008, 20: 977-988

33 Krompinger JW, Moser JS, Simons RF. Modulations of the Electrophysiological Response to Pleasant Stimuli by Cognitive Reappraisal. Emotion, 2008, 8: 132-137

34 Moser JS, Hajcak G, Eukay E, Simons RF. Intentional modulation of emotional responding to unpleasant pictures: An ERP study. Psychophysiology, 2006, 43: 292-296

35 Schupp HT, Junghöfer M, Weike AI, Hamm AO. The selective processing of briefly presented affective pictures: An ERP analysis. Psychophysiology, 2004, 41: 441-449

36 Cuthbert BN, Schupp HT, Bradley MM, Birbaumer N, Lang PJ. Brain potentials in affective picture processing: Covariation with autonomic arousal and affective report. Biol Psychol, 2000, 52: 95-111

37 Gross JJ, John OP. Individual differences in two emotion regulation processes: Implications for affect, relationships, and well-being. J Pers Soc Psychol, 2003, 85: 348-362

38 Lang PJ, Bradley MM, Cuthbert BN. International Affective Picture System (IAPS): Technical manual and affective ratings. NIMH Center for the Study of Emotion and Attention, 1997

39 Bai L, Ma H, Luo YJ. The development of native Chinese affective picture system. Chinese Mental Health Journal, 2005, 19: 719-712

40 Huang YX, Luo YJ. Native assessment of international affective picture system. Chinese Mental Health Journal, 2004, 9: 631-634

41 Winecoff A, Clithero JA, Carter RM, Bergman SR, Wang L, Huettel SA. Ventromedial Prefrontal Cortex Encodes Emotional Value. J Neurosci, 2013, 33: 11032-11039

42 Luck SJ. An introduction to event-related potentials and their neural origins. In: Luch SJ, editor. An Introduction to the Event-Related Potential Technique. Cambridge, MA: MIT, 2005: 107

43 Carretié L, Mercado F, Tapia M, Hinojosa JA. Emotion, attention, and the 'negativity bias', studied through event-related potentials. Int J Psychophysiol, 2001,41: 75-85
44 Yuan JJ, He YY, Lei Y, Yang JM, Li H. Event-Related Potential correlates of the extraverts' sensitivity to valence changes in positive stimuli. NeuroReport, 2009, 12: 1071-1076

45 Del Cul A, Baillet S, Dehaene S. Brain dynamics underlying the nonlinear threshold for access to consciousness. PLoS Biol, 2007, 5: $1-16$

46 Carretié L, Hinojosa JA, Martín-Loeches M, Mercado F, Tapia M. Automatic attention to emotional stimuli: neural correlates. Hum brain map, 2004, 22: 290-299

47 Saito M, Ishida T. Cognitive resource model for the information-processing of task-irrelevant visual stimuli. Psychiatry Clin Neurosci, 2002, 56: 145-151

48 Schupp HT, Cuthbert BN, Bradley MM, Cacioppo JT, Ito T, Lang PJ. Affective picture processing: The late positive potential is modulated by motivational relevance. Psychophysiology, 2000, 37: 257-261

49 Friesen WV. Cultural differences in facial expressions in a social situation: An experimental test of the concept of display rules. Unpublished doctoral dissertation, University of California, San Francisco, 1972

50 Matsumoto D. Individual and cultural differences in status differentiation: The Status Differentiation Scale. Journal of Cross-Cultural Psychology, 2007, 38: 413-431

51 Suh E, Diener E, Oishi S, Triandis HC. The shifting basis of life satisfaction judgments across cultures: Emotions versus norms. J Pers Soc Psychol, 1998, 74: 482-493

52 Carretié L, Hinojosa JA, Mercado F. Cerebral patterns of attentional habituation to emotional visual stimuli. Psychophysiology, 2003, 40: 381-388

53 Labus JS., Naliboff BD, Berman SM, Suyenobu B, Vianna EP, Tillisch K, Mayer EA. Brain networks underlying perceptual habituation to repeated aversive visceral stimuli in patients with irritable bowel syndrome. Neuroimage, 2009, 47: 952-960

54 Holt DJ, Weiss AP, Rauch SL, Wright CI, Zalesak, M Goff, DC, Ditman T, Welsh RC, Heckers S. Sustained activation of the hippocampus in response to fearful faces in schizophrenia. Biol psychiatry, 2005, 57: 1011-1019

55 Smith NK, Larsen JT, Chartrand TL, Cacioppo JT, Katafiasz HA, Moran KE. Being bad isn't always good: Affective context moderates the attention bias toward negative information. J Pers Soc Psychol, 2006, 90: 210-220

56 Yuan J, Chen J, Yang J, Ju E, Norman GJ, Ding, NX. Negative Mood State Enhances the Susceptibility to Unpleasant Events: Neural Correlates from a Music-Primed Emotion Classification Task. PLoS ONE, 2010, 9: e89844. doi:10.1371/journal.pone.0089844

Open Access This article is distributed under the terms of the Creative Commons Attribution License which permits any use, distribution, and reproduction in any medium, provided the original author(s) and source are credited. 